\title{
MONODROMY REPRESENTATIONS OF HYPERGEOMETRIC SYSTEMS WITH RESPECT TO FUNDAMENTAL SERIES SOLUTIONS
}

\author{
KEIJI MATSUMOTO
}

\begin{abstract}
We study the monodromy representation of the generalized hypergeometric differential equation and that of Lauricella's $F_{C}$ system of hypergeometric differential equations. We use fundamental systems of solutions expressed by the hypergeometric series. We express non-diagonal circuit matrices as reflections with respect to root vectors with all entries 1 . We present a simple way to obtain circuit matrices.
\end{abstract}

\section{InTRODUCTION}

The hypergeometric series ${ }_{2} F_{1}\left(\begin{array}{c}a_{1}, a_{2} \\ b_{1}\end{array} ; x\right)$ satisfies the hypergeometric differential equation, which is second order linear, and with regular singular points at $x=0,1, \infty$. There are two natural ways to generalize the hypergeometric differential equations: one is to higher rank ordinary differential equations and the other is to integrable systems of differential equations of multi-variables. As the former, generalized hypergeometric series and equations are well known. As the latter, four kinds of hypergeometric series and systems of hypergeometric differential equations are introduced by P. Appell and G. Lauricella.

In this paper, we study the monodromy representation of the generalized hypergeometric differential equation and that of Lauricella's $F_{C}$ system of hypergeometric differential equations. We use fundamental systems of solutions expressed by hypergeometric series. We express the circuit matrices along generators of the fundamental group of the complement of the singular locus with respect to each fundamental system of solutions. The aim of this paper is the presentation of a simple way to obtain circuit matrices.

Let us explain our method. For each case of the study of monodromy representations, the problem reduces to determining a circuit matrix

Date: June 20, 2018.

2010 Mathematics Subject Classification. 32S40, 58K10, 34M35, 33C20, 33C65.

Key words and phrases. Monodromy representation, hyperegeometric functions. 
$M$, since the others are trivially given as diagonal matrices. We can regard this target circuit matrix $M$ as a complex reflection with respect to a kind of an inner product, i.e., the eigenspace of $M$ of eigenvalue 1 is the orthogonal complement of an eigenvector $v$ of $M$ of eigenvalue $l(\neq 1)$. Let $H$ be the gram matrix of our fundamental system of solutions with respect to this inner product. We can show that it is diagonal. We normalize our fundamental system so that the $l$-eigenvector $v$ of $M$ becomes $(1, \ldots, 1)$. Though the matrix $H$ is changed by this normalization, it is still diagonal. By regarding diagonal entries of $H$ as indeterminants, we set up a system of equations by the Riemann scheme or the relations induced from the fundamental group. By solving it, we determine the matrices $H$ and $M$.

There are several studies for the monodromy representation of the generalized hypergeometric differential equation, refer to [BH], [Le], [Mi], and [O]. For that of Lauricella's $F_{C}$ system in two variables, we have many ways to compute circuit matrices, refer to [GM], [HU], [Kan, [Kat and [T]. The case of $m$ variables, it was an open problem for a long time to determine the monodromy representation. We did not have a simple system of generators of the fundamental group of the complement of the singular locus. Recently, this open problem is solved in [G]: it is shown that the fundamental group is generated by $m+1$ loops, and that the circuit transformations along them can be expressed by the intersection from on twisted homology groups associated with Euler type integral representations of solutions.

This paper consists of four sections. We determine the monodromy representations of the hypergeometric differential equation, of generalized one, and of Lauricella's $F_{C}$ system in $\S 2, \S 3$ and $\S 4$, respectively. We can obtain the results in $\S 2$ from those in $\S 3$ by regarding the rank $p$ as 2. However, we describe details in $\S 2$ since this section helps readers to understand our method well, and results in $\S 2$ need when we prove the key proposition in $\S 4$ by the induction on the number of variables. Our study in $\S 4$ is based on some results in G. Lemma 4.1 is an addition to them associated with the fundamental group. This lemma relates a product of loops in $\mathbb{C}^{m}$ to a loop in $\mathbb{C}^{m-1}$, and enables us to decrease the number of variables. Anyone can simply give an expression of the circuit matrix $M$ for the case of two variables by the reduction to results in $\S 2$. 


\section{Monodromy Representation OF ${ }_{2} F_{1}$}

2.1. Hypergeometric differential equation. The hypergeometric series ${ }_{2} F_{1}\left(\begin{array}{c}a_{1}, a_{2} \\ b_{1}\end{array} ; x\right)$ is defined by

$$
{ }_{2} F_{1}\left(\begin{array}{c}
a_{1}, a_{2} \\
b_{1}
\end{array} ; x\right)=\sum_{n=0}^{\infty} \frac{\left(a_{1}, n\right)\left(a_{2}, n\right)}{\left(b_{1}, n\right)(1, n)} x^{n},
$$

where the main variable $x$ is in $\{x \in \mathbb{C}|| x \mid<1\}, a_{1}, a_{2}, b_{1}$ are complex parameters with $b_{1} \notin-\mathbb{N}=\{0,-1,-2, \ldots\}$, and Pochhammer's symbol $(a, n)$ stands for $a(a+1) \cdots(a+n-1)$. This function satisfies the hypergeometric differential equation

$$
\left[x(1-x)\left(\frac{d}{d x}\right)^{2}+\left\{b_{1}-\left(a_{1}+a_{2}+1\right) x\right\}\left(\frac{d}{d x}\right)-a_{1} a_{2}\right] f(x)=0 .
$$

This is a Fuchsian differential equation with regular singular points at $x=0,1, \infty$. The Riemann scheme of (1) is

$$
\begin{array}{ccc}
x=0 & x=1 & x=\infty \\
\hline 0 & 0 & a_{1} \\
1-b_{1} & b_{1}-a_{1}-a_{2} & a_{2}
\end{array}
$$

and a fundamental system of solutions to (11) for $b_{1} \notin \mathbb{Z}$ around $\dot{x}=\varepsilon$ is given by the column vector

$$
\left(\begin{array}{c}
{ }_{2} F_{1}\left(\begin{array}{c}
a_{1}, a_{2} \\
b_{1} ; x
\end{array}\right) \\
x^{1-b_{1}}{ }_{2} F_{1}\left(\begin{array}{c}
a_{1}-b_{1}+1, a_{2}-b_{1}+1 \\
2-b_{1}
\end{array} ; x\right)
\end{array}\right),
$$

where $\varepsilon$ is a sufficiently small positive real number.

2.2. Circuit matrices $M_{0}$ and $M_{1}$. In this subsection, we assume that

$$
a_{1}, a_{2}, b_{1}, a_{1}-b_{1}, a_{2}-b_{1}, a_{1}+a_{2}-b_{1} \notin \mathbb{Z} .
$$

We set

$$
A_{1}=\exp \left(2 \pi \sqrt{-1} a_{1}\right), \quad A_{2}=\exp \left(2 \pi \sqrt{-1} a_{2}\right), \quad B_{1}=\exp \left(2 \pi \sqrt{-1} b_{1}\right),
$$

which are different from 1 under our assumption. Let $\rho_{0}$ and $\rho_{1}$ be loops in $X=\mathbb{C}-\{0,1\}$ with base $\dot{x}=\varepsilon$ represented by

$$
\begin{aligned}
& \rho_{0}: \quad[0,1] \ni t \mapsto \varepsilon e^{2 \pi \sqrt{-1} t} \in X, \\
& \rho_{1}: \quad[0,1] \ni t \mapsto 1-(1-\varepsilon) e^{2 \pi \sqrt{-1} t} \in X .
\end{aligned}
$$

Note that $\rho_{0}$ and $\rho_{1}$ turn positively around $x=0$ and $x=1$ once, respectively. The fundamental group $\pi_{1}(X, \dot{x})$ is freely generated by 
these loops. We set $\rho_{\infty}=\left(\rho_{0} \circ \rho_{1}\right)^{-1}$, where $\rho_{0} \circ \rho_{1}$ is a loop joining $\rho_{0}$ to $\rho_{1}$.

We select a fundamental system of solutions to (1) around $\dot{x}$ as

$$
\mathbf{F}_{2}^{g}(x)=\left(\begin{array}{cc}
g_{1} & 0 \\
0 & g_{2}
\end{array}\right)\left(\begin{array}{c}
{ }_{2} F_{1}\left(\begin{array}{c}
a_{1}, a_{2} \\
b_{1} ; x
\end{array}\right) \\
x^{1-b_{1}} F_{1}\left(\begin{array}{c}
a_{1}-b_{1}+1, a_{2}-b_{1}+1 \\
2-b_{1}
\end{array} ; x\right)
\end{array}\right),
$$

where $g_{1}$ and $g_{2}$ are non-zero constants. Let $\rho$ be an element of $\pi_{1}(X, \dot{x})$. Then there exists $M_{\rho} \in G L_{2}(\mathbb{C})$ such that the analytic continuation of $\mathbf{F}_{2}^{g}(x)$ along $\rho$ is expressed as

$$
M_{\rho}^{g} \mathbf{F}_{2}^{g}(x) .
$$

We call $M_{\rho}^{g}$ the circuit matrix along $\rho$ with respect to the basis $\mathbf{F}_{2}^{g}(x)$. We set

$$
M_{0}^{g}=M_{\rho_{0}}^{g}, \quad M_{1}^{g}=M_{\rho_{1}}^{g}, \quad M_{\infty}^{g}=M_{\rho_{\infty}}^{g} .
$$

By the expression of $\mathbf{F}_{2}^{g}(x)$, the following is obvious.

Lemma 2.1. For any non-zero constants $g_{1}$ and $g_{2}$, we have

$$
M_{0}^{g}=\left(\begin{array}{cc}
1 & 0 \\
0 & B_{1}^{-1}
\end{array}\right)
$$

By using an Euler type integral representation of solutions to (1), we can show the following as is in Lemma 5.2 of [Ma].

Lemma 2.2. There exists $H \in G L_{2}(\mathbb{C})$ such that

$$
M_{\rho}^{g} H^{t}\left(M_{\rho}^{g}\right)^{\vee}=H
$$

for any $\rho \in \pi_{1}(X, \dot{x})$, where $z\left(a_{1}, a_{2}, b_{1}\right)^{\vee}=z\left(-a_{1},-a_{2},-b_{1}\right)$ for any function $z$ of $a_{1}, a_{2}, b_{1}$, and $Z^{\vee}=\left(z_{i j}^{\vee}\right)$ for a matrix $Z=\left(z_{i j}\right)$.

Note that the matrix $H$ depends on the ratio of $g_{1}$ and $g_{2}$. We treat the entries of $H$ as indeterminants. By determining them, we express a representation matrix of the circuit transformation along $\rho_{1}$.

Lemma 2.3. The matrix $H$ in Lemma 2.2 is diagonal.

Proof. We set $H=\left(\begin{array}{ll}h_{11} & h_{12} \\ h_{21} & h_{22}\end{array}\right)$. By Lemma 2.2, we have

$$
\begin{aligned}
M_{0}^{g} H^{t}\left(M_{0}^{g}\right)^{\vee} & =\left(\begin{array}{cc}
1 & 0 \\
0 & B_{1}^{-1}
\end{array}\right)\left(\begin{array}{ll}
h_{11} & h_{12} \\
h_{21} & h_{22}
\end{array}\right)\left(\begin{array}{cc}
1 & 0 \\
0 & B_{1}
\end{array}\right) \\
& =\left(\begin{array}{cc}
h_{11} & B_{1} h_{12} \\
B_{1}^{-1} h_{21} & h_{22}
\end{array}\right)=H .
\end{aligned}
$$

Since $B_{1} \neq 1$ under our assumption, $h_{12}$ and $h_{21}$ should be 0 . 
By the Riemann scheme (2), it is easy to see that the eigenvalues of $M_{1}$ are 1 and $l=B_{1} /\left(A_{1} A_{2}\right)$. Note that $l \neq 1$ under our assumption.

Lemma 2.4. Let $v=\left(v_{1}, v_{2}\right)$ be the eigenvector of $M_{1}^{g}$ of eigenvalue $l=B_{1} /\left(A_{1} A_{2}\right)$, and $w$ be that of eigenvalue 1 . Then we have

$$
w H^{t} v^{\vee}=0, \quad v H^{t} v^{\vee} \neq 0, \quad v_{1} v_{2} \neq 0 .
$$

Proof. By Lemma 2.2, we have

$$
\begin{aligned}
w H^{t} v^{\vee} & =w\left(M_{1}^{g} H^{t}\left(M_{1}^{g}\right)^{\vee}\right)^{t} v^{\vee}=\left(w M_{1}^{g}\right) H^{t}\left(v M_{1}^{g}\right)^{\vee} \\
& =l w H^{t} v^{\vee} .
\end{aligned}
$$

Since $l \neq 1, w H^{t} v^{\vee}$ vanishes.

Note that

$$
\left(\begin{array}{c}
v \\
w
\end{array}\right) H^{t}\left(\begin{array}{c}
v \\
w
\end{array}\right)^{\vee}=\left(\begin{array}{cc}
v H^{t} v^{\vee} & 0 \\
0 & w H^{t} w^{\vee}
\end{array}\right) .
$$

Since $v$ and $w$ are linearly independent, if $v H^{t} v^{\vee}=0$ then $H$ degenerates. This contradicts to $H \in G L_{2}(\mathbb{C})$. Thus we have $v H^{t} v^{\vee} \neq 0$.

Suppose that $v_{1}=0$. Then $(0,1)$ is the eigen vector of $M_{1}^{g}$ of eigenvalue $l$. By the equality $w H^{t} v^{\vee}=0,(1,0)$ is the eigen vector of $M_{1}^{g}$ of eigenvalue 1 . Thus we have

$$
M_{1}^{g}=\left(\begin{array}{cc}
1 & 0 \\
0 & B_{1} /\left(A_{1} A_{2}\right)
\end{array}\right) .
$$

The eigenvalues of

$$
\left(M_{\infty}^{g}\right)^{-1}=M_{0}^{g} M_{1}^{g}=\left(\begin{array}{cc}
1 & 0 \\
0 & 1 /\left(A_{1} A_{2}\right)
\end{array}\right)
$$

are 1 and $1 /\left(A_{1} A_{2}\right)$; this contradicts to the Riemann scheme (2) under our assumption (31). Hence we have $v_{1} \neq 0$. We can similarly show $v_{2} \neq 0$.

Note that the eigenvector $v$ of $M_{1}^{g}$ of eigenvalue $l$ depends on the ratio of $g_{1}$ and $g_{2}$. We can choose $g_{1}, g_{2}$ in (5) so that the eigenvector $v$ of $M_{1}^{g}$ of eigenvalue $l$ becomes $\mathrm{v}=(1,1)$. From now on, we fix the constants $g_{1}$ and $g_{2}$ as the above values. We denote the fundamental system of solutions to (11) around $\dot{x}$ for these constants in (5) by $\mathbf{F}_{2}(x)$. The circuit matrices along $\rho_{0}, \rho_{1}, \rho_{\infty}$ with respect to $\mathbf{F}_{2}(x)$ are denoted by $M_{0}, M_{1}, M_{\infty}$, respectively.

Lemma 2.5. The circuit matrix $M_{1}$ is expressed as

$$
M_{1}=i d_{2}-\frac{1-l}{\mathrm{vH}^{t} \mathrm{v}} H^{t} \mathrm{vv}
$$

where $i d_{m}$ is the unit matrix of size $m, l=B_{1} /\left(A_{1} A_{2}\right)$ and $\mathrm{v}=(1,1)$. 
Proof. We set

$$
M_{1}^{\prime}=i d_{2}-\frac{1-l}{\mathrm{v} H^{t} \mathrm{v}} H^{t} \mathrm{vv} .
$$

We show that the eigenspaces of $M_{1}^{\prime}$ coincides with those of $M_{1}$. We have

$$
\begin{aligned}
\mathrm{v}_{1}^{\prime} & =\mathrm{v}\left(i d_{2}-\frac{1-l}{\mathrm{v}^{t}{ }_{\mathrm{v}}} H^{t} \mathrm{vv}\right) \\
& =\mathrm{v}-(1-l) \mathrm{v}=l \mathrm{v},
\end{aligned}
$$

which means $\mathrm{v}$ is an eigenvector of $M_{1}^{\prime}$ of eigenvalue $l$. Let $w$ be a vector satisfying $w H^{t} \mathrm{v}=0$. Then we have

$$
\begin{aligned}
w M_{1}^{\prime} & =w\left(i d_{2}-\frac{1-l}{\mathrm{v} H^{t} \mathrm{v}} H^{t} \mathrm{vv}\right) \\
& =w-\frac{(1-l) w H^{t} \mathrm{v}}{\mathrm{v} H^{t} \mathrm{v}} \mathrm{v}=w
\end{aligned}
$$

which means $w$ is an eigenvector of $M_{1}^{\prime}$ of eigenvalue 1 . Since $M_{1}$ and $M_{1}^{\prime}$ have the same eigenspaces, they coincide as matrices.

We regard the diagonal entries of $H$ as indeterminants in the expression of $M_{1}$ in Lemma 2.5. By evaluating them, we determine the circuit matrix $M_{1}$. Note that the expression of $M_{1}$ in Lemma 2.5] is invariant under a scalar multiple to $H$. We can assume that

$$
H=\left(\begin{array}{ll}
1 & 0 \\
0 & h
\end{array}\right)
$$

Proposition 2.1. We have

$$
\begin{aligned}
& h=-\frac{\left(B_{1}-A_{1}\right)\left(B_{1}-A_{2}\right)}{B_{1}\left(A_{1}-1\right)\left(A_{2}-1\right)} \\
& M_{1}=i d_{2}-\left(\begin{array}{cc}
\frac{B_{1}\left(A_{1}-1\right)\left(A_{2}-1\right)}{A_{1} A_{2}\left(B_{1}-1\right)} & \frac{B_{1}\left(A_{1}-1\right)\left(A_{2}-1\right)}{A_{1} A_{2}\left(B_{1}-1\right)} \\
\frac{\left(B_{1}-A_{1}\right)\left(B_{1}-A_{2}\right)}{A_{1} A_{2}\left(B_{1}-1\right)} & \frac{\left(B_{1}-A_{1}\right)\left(B_{1}-A_{2}\right)}{A_{1} A_{2}\left(B_{1}-1\right)}
\end{array}\right) .
\end{aligned}
$$

Proof. We compute the trace of $M_{0} M_{1}$, which should be $1 / A_{1}+1 / A_{2}$ by the Riemann scheme (2). Since

$$
M_{0} M_{1}=\left(\begin{array}{cc}
1 & 0 \\
0 & B_{1}^{-1}
\end{array}\right)-\frac{1-l}{1+h}\left(\begin{array}{cc}
1 & 1 \\
B_{1}^{-1} h & B_{1}^{-1} h
\end{array}\right)
$$


we have

$$
\begin{aligned}
\operatorname{tr}\left(M_{0} M_{1}\right) & =1+B_{1}^{-1}+\frac{(l-1)\left(1+B_{1}^{-1} h\right)}{1+h} \\
& =\frac{\left(A_{1} A_{2}+1\right) B_{1} h+A_{1} A_{2}+B_{1}^{2}}{A_{1} A_{2} B_{1}(1+h)}=\frac{1}{A_{1}}+\frac{1}{A_{2}} .
\end{aligned}
$$

We can reduce the last equation to a linear equation with respect to $h$, which is solved as

$$
h=-\frac{\left(A_{1}-B_{1}\right)\left(A_{2}-B_{1}\right)}{B_{1}\left(A_{1}-1\right)\left(A_{2}-1\right)} .
$$

We obtain the expression of $M_{1}$ by the substitution of this solution into Lemma 2.5.

Remark 2.1. Note that

$$
\mathrm{v} H^{t} \mathrm{v}=\operatorname{tr}(H)=\frac{\left(A_{1} A_{2}-B_{1}\right)\left(B_{1}-1\right)}{\left(A_{1}-1\right)\left(A_{2}-1\right) B_{1}} .
$$

We have

$$
\frac{1-l}{\mathrm{v}^{t}{ }^{t} \mathrm{~V}}=\frac{A_{1} A_{2}-B_{1}}{A_{1} A_{2}} \times \frac{\left(A_{1}-1\right)\left(A_{2}-1\right) B_{1}}{\left(A_{1} A_{2}-B_{1}\right)\left(B_{1}-1\right)}=\frac{\left(A_{1}-1\right)\left(A_{2}-1\right) B_{1}}{A_{1} A_{2}\left(B_{1}-1\right)},
$$

in which the factor $A_{1} A_{2}-B_{1}$ is canceled.

We conclude this subsection by the following.

Theorem 2.1. Suppose the non-integral condition (3) for $a_{1}, a_{2}$ and $b_{1}$. Then there exists a fundamental system $\mathbf{F}_{2}(x)$ of solutions to the hypergeometric differential equation (1) around $\dot{x}=\varepsilon$ such that the circuit matrix $M_{0}$ and $M_{1}$ along the loops $\rho_{0}$ and $\rho_{1}$ in (4) are expressed as

$$
M_{0}=\left(\begin{array}{cc}
1 & 0 \\
0 & B_{1}^{-1}
\end{array}\right), \quad M_{1}=i d_{2}-\frac{1-l}{\mathrm{v} H^{t} \mathrm{v}} H^{t} \mathrm{vv}
$$

where $\varepsilon$ is a sufficiently small positive real numbers, $A_{1}=e^{2 \pi \sqrt{-1} a_{1}}$, $A_{2}=e^{2 \pi \sqrt{-1} a_{2}}, B_{1}=e^{2 \pi \sqrt{-1} b_{1}}, l=B_{1} /\left(A_{1} A_{2}\right), \mathrm{v}=(1,1)$ and

$$
H=\left(\begin{array}{cc}
1 & 0 \\
0 & -\frac{\left(A_{1}-B_{1}\right)\left(A_{2}-B_{1}\right)}{B_{1}\left(A_{1}-1\right)\left(A_{2}-1\right)}
\end{array}\right) .
$$




\section{Monodromy Representation of ${ }_{p} F_{p-1}$}

3.1. Generalized hypergeometric differential equation. The generalized hypergeometric series is defined by

$$
{ }_{p} F_{p-1}\left(\begin{array}{c}
a_{1}, \ldots, a_{p} \\
b_{1} \ldots, b_{p-1}
\end{array} ;\right)=\sum_{n=0}^{\infty} \frac{\left(a_{1}, n\right) \cdots\left(a_{p}, n\right)}{\left(b_{1}, n\right) \cdots\left(b_{p-1}, n\right)(1, n)} x^{n},
$$

where the main variable $x$ is in $\{x \in \mathbb{C}|| x \mid<1\}, a_{1}, \ldots, a_{p}, b_{1}, \ldots, b_{p-1}$ are complex parameters with $b_{1}, \ldots, b_{p-1} \notin-\mathbb{N}$. This series satisfies the differential equation of rank $p$ :

$$
\begin{aligned}
& \left(x \frac{d}{d x}+a_{1}\right) \cdots\left(x \frac{d}{d x}+a_{p}\right) f(x) \\
= & \frac{d}{d x}\left(x \frac{d}{d x}+b_{1}-1\right) \cdots\left(x \frac{d}{d x}+b_{p-1}-1\right) f(x) .
\end{aligned}
$$

This is a Fuchsian differential equation with regular singular points at $x=0,1, \infty$. The Riemann scheme of (6) is

\begin{tabular}{ccc}
$x=0$ & $x=1$ & $x=\infty$ \\
\hline 0 & 0 & $a_{1}$ \\
$1-b_{1}$ & 1 & $a_{2}$ \\
$\vdots$ & $\vdots$ & $\vdots$ \\
$1-b_{p-2}$ & $p-2$ & $a_{p-1}$ \\
$1-b_{p-1}$ & $\sum_{j=1}^{p-1} b_{j}-\sum_{i=1}^{p} a_{i}$ & $a_{p}$
\end{tabular}

and a fundamental system of solutions to (6) for $b_{1}, \ldots, b_{p-1} \notin \mathbb{Z}$ around $\dot{x}=\varepsilon$ is given by

$$
\left.\left(\begin{array}{c}
{ }_{p} F_{p-1}\left(\begin{array}{c}
\left.a_{1}, \ldots, a_{p} ; x\right) \\
b_{1}, \ldots, b_{p-1}
\end{array}\right) \\
x^{1-b_{1}{ }_{p} F_{p-1}}\left(\begin{array}{c}
a_{1}-b_{1}+1, \ldots, a_{p}-b_{1}+1 \\
2-b_{1}, b_{2}-b_{1}+1, \ldots, b_{p-1}-b_{1}+1
\end{array} ; x\right) \\
\vdots \\
x^{1-b_{p-1} F_{p-1}}\left(\begin{array}{c}
a_{1}-b_{p-1}+1, \ldots, a_{p}-b_{p-1}+1 \\
b_{1}-b_{p-1}+1, \ldots, b_{p-2}-b_{p-1}+1,2-b_{p-1}
\end{array} ; x\right.
\end{array}\right)\right)
$$

where $\varepsilon$ is a sufficiently small positive real number. Note that there are $p-1$ linearly independent holomorphic solutions to (6) on an annulus $\{x \in \mathbb{C}|0<| x-1 \mid<\varepsilon\}$.

3.2. Circuit matrices $M_{0}$ and $M_{1}$. In this subsection, we assume that

$$
a_{i}, b_{j}, a_{i}-b_{j}, b_{j}-b_{j^{\prime}}, \sum_{i=1}^{p} a_{i}-\sum_{j=1}^{p-1} b_{j} \notin \mathbb{Z}
$$


where $1 \leq i \leq p, 1 \leq j, j^{\prime} \leq p-1$ and $j \neq j^{\prime}$. We set

$$
A_{i}=\exp \left(2 \pi \sqrt{-1} a_{i}\right), \quad B_{j}=\exp \left(2 \pi \sqrt{-1} b_{j}\right)
$$

for $1 \leq i \leq p$ and $1 \leq j \leq p-1$. We choose a fundamental system $\mathbf{F}_{p}^{g}(x)$ of solutions to (6) around $\dot{x}=\varepsilon$ as the left multiplication of the diagonal matrix

$$
g=\left(\begin{array}{llll}
g_{1} & & & \\
& g_{2} & & \\
& & \ddots & \\
& & & g_{p}
\end{array}\right) \in G L_{p}(\mathbb{C})
$$

to the column vector (8).

Let $M_{0}^{g}$ and $M_{1}^{g}$ be the circuit matrices along the loops $\rho_{0}$ and $\rho_{1}$ in (4) with respect to $\mathbf{F}_{p}^{g}(x)$. We set $M_{\infty}^{g}=\left(M_{0}^{g} M_{1}^{g}\right)^{-1}$.

Lemma 3.1. For any diagonal matrix $g \in G L_{p}(\mathbb{C})$, the circuit matrix $M_{0}^{g}$ is

$$
\left(\begin{array}{llll}
1 & & & \\
& B_{1}^{-1} & & \\
& & \ddots & \\
& & & B_{p-1}^{-1}
\end{array}\right) .
$$

Proof. It is clear by (8) .

As is in subsection 2.2, we have the following lemma.

Lemma 3.2. Let $M_{\rho}^{g}$ be the circuit matrix along $\rho \in \pi_{1}(X, \dot{x})$ with respect to $\mathbf{F}_{p}^{g}(x)$. Then there exists a diagonal matrix $H \in G L_{p}(\mathbb{C})$ such that

$$
M_{\rho}^{g} H^{t}\left(M_{\rho}^{g}\right)^{\vee}=H,
$$

where $z\left(a_{1}, \ldots, a_{p}, b_{1}, \ldots, b_{p-1}\right)^{\vee}=z\left(-a_{1}, \ldots,-a_{p},-b_{1}, \ldots,-b_{p-1}\right)$ for any function $z$ of the parameters.

The matrix $H$ depends on the ratio of $g_{1}$ and $g_{2}$. We treat the entries of $H$ as indeterminants.

By the Riemann scheme (7) and our assumption (9), the eigenvalues of $M_{1}^{g}$ are 1 and

$$
l=\left(\prod_{j=1}^{p-1} B_{j}\right) /\left(\prod_{i=1}^{p} A_{i}\right)
$$

the eigenspace of $M_{1}^{g}$ of eigenvalue 1 is $p-1$ dimensional and that of eigenvalue $l$ is one dimensional. 
Lemma 3.3. Let $v=\left(v_{1}, \ldots, v_{p}\right)$ be an eigenvector of $M_{1}^{g}$ of eigenvalue l. Then the eigenspace of $M_{1}^{g}$ of eigenvalue 1 is characterized as

$$
\left\{w \in \mathbb{C}^{p} \mid w H^{t} v^{\vee}=0\right\} .
$$

Moreover, the vector $v$ satisfies

$$
v H^{t} v^{\vee} \neq 0 \text {. }
$$

Proof. Trace the proof of Lemma 2.4.

Lemma 3.4. Let $v=\left(v_{1}, \ldots, v_{p}\right)$ be an eigenvector of $M_{1}^{g}$ of eigenvalue l. Then the circuit matrix $M_{1}^{g}$ is expressed as

$$
M_{1}^{g}=i d_{p}-\frac{1-l}{v H^{t} v^{\vee}} H^{t} v^{\vee} v
$$

Moreover, none of $v_{1}, \ldots, v_{p}$ vanishes.

Proof. We set

$$
M_{1}^{\prime}=i d_{p}-\frac{1-l}{v H^{t} v^{\vee}} H^{t} v^{\vee} v .
$$

We show that the eigenspaces of $M_{1}^{\prime}$ coincides with those of $M_{1}^{g}$. Note that

$$
\begin{aligned}
& v M_{1}^{\prime}=v\left(i d_{p}-\frac{1-l}{v H^{t} v^{\vee}} H^{t} v^{\vee} v\right)=v-(1-l) v=l v \\
& w M_{1}^{\prime}=w\left(i d_{p}-\frac{1-l}{v H^{t} v^{\vee}} H^{t} v^{\vee} v\right)=w-\frac{(1-l) w H^{t} v^{\vee}}{v H^{t} v^{\vee}} v=w
\end{aligned}
$$

for any element $w$ satisfying $w H^{t} v^{\vee}=0$. By Lemma 3.3, we have $M_{1}^{\prime}=M_{1}^{g}$.

Suppose that $v_{i}=0$. Then the matrix $M_{1}^{g}$ takes the form

$$
i\left(\begin{array}{ccc}
* & i & \\
{ }^{t} \mathbf{0} & * \\
\mathbf{0} & 1 & \mathbf{0}^{\prime} \\
* & { }^{t} \mathbf{0}^{\prime} & *
\end{array}\right)
$$

by its expression, where $\mathbf{0}$ and $\mathbf{0}^{\prime}$ are zero vectors. Since $M_{0}^{g}$ is diagonal, we have

$$
M_{0}^{g} M_{1}^{g}=i\left(\begin{array}{ccc}
* & i & \\
& { }^{t} \mathbf{0} & * \\
\mathbf{0} & B_{i-1}^{-1} & \mathbf{0}^{\prime} \\
* & { }^{t} \mathbf{0}^{\prime} & *
\end{array}\right),
$$

where we regard $B_{0}$ as 1 . Hence $M_{\infty}$ has an eigenvalue $B_{i-1}$, which contradicts to the Riemann scheme (7) under our assumption (91). Therefore, we have $v_{i} \neq 0$ for $1 \leq i \leq p$. 
We choose $g_{1}, \ldots, g_{p}$ so that the eigenvector of eigenvalue $l$ becomes $\mathrm{v}=(1, \ldots, 1)$. From now on, we fix the constants $g_{1}, \ldots, g_{p}$ as the above values. We denote the fundamental system of solutions to (6) around $\dot{x}$ for these constants in $\mathbf{F}_{p}^{g}(x)$ by $\mathbf{F}_{p}(x)$. The circuit matrices with respect to $\mathbf{F}_{p}(x)$ are expressed by

$$
M_{0}=\left(\begin{array}{cccc}
1 & & & \\
& B_{1}^{-1} & & \\
& & \ddots & \\
& & & B_{p-1}^{-1}
\end{array}\right), \quad M_{1}=i d_{p}-\frac{1-l}{\mathrm{v} H^{t} \mathrm{v}} H^{t} \mathrm{vv}
$$

Here we regard the diagonal entries of $H$ as indeterminants in the expression of $M_{1}$. By evaluating them, we determine the expression of $M_{1}$. Note that the expression of $M_{1}$ is invariant under a scalar multiple to $H$. We can assume that

$$
H=\left(\begin{array}{cccc}
1 & & & \\
& h_{1} & & \\
& & \ddots & \\
& & & h_{p-1}
\end{array}\right)
$$

Note that the matrix $H$ is unique after this normalization.

Proposition 3.1. For $1 \leq k \leq p-1$, we have

$$
h_{k}=\frac{-\left(\prod_{1 \leq j \leq p-1}^{j \neq k}\left(B_{j}-1\right)\right)\left(\prod_{i=1}^{p}\left(A_{i}-B_{k}\right)\right)}{B_{k}\left(\prod_{1 \leq j \leq p-1}^{j \neq k}\left(B_{j}-B_{k}\right)\right)\left(\prod_{i=1}^{p}\left(A_{i}-1\right)\right)} .
$$

Proof. We consider the eigen polynomial

$$
Q(t)=\operatorname{det}\left(t \cdot i d_{p}-M_{0} M_{1}\right)
$$


of the matrix $M_{0} M_{1}=M_{\infty}^{-1}$. By the Riemann scheme (17) $1 / A_{1}, \ldots, 1 / A_{p}$ are solutions to the equation $Q(t)=0$. Thus we have

$$
\begin{aligned}
& \operatorname{det}\left(M_{0} M_{1}-i d_{p} / A_{\ell}\right) \\
& =\left|\begin{array}{ccccc}
d_{0}+\mu & \mu & \mu & \cdots & \mu \\
\mu B_{1}^{-1} h_{1} & d_{1}+\mu B_{1}^{-1} h_{1} & \mu B_{1}^{-1} h_{1} & \cdots & \mu B_{1}^{-1} h_{1} \\
\mu B_{2}^{-1} h_{2} & \mu B_{2}^{-1} h_{2} & d_{2}+\mu B_{2}^{-1} h_{2} & \cdots & \mu B_{2}^{-1} h_{2} \\
\vdots & \vdots & \vdots & \ddots & \vdots \\
\mu B_{p-1}^{-1} h_{p-1} & \mu B_{p-1}^{-1} h_{p-1} & \mu B_{p-1}^{-1} h_{p-1} & \cdots & d_{p-1}+\mu B_{p-1}^{-1} h_{p-1}
\end{array}\right| \\
& =\left|\begin{array}{ccccc}
d_{0}+\mu & \mu & \mu & \cdots & \mu \\
-d_{0} B_{1}^{-1} h_{1} & d_{1} & 0 & \cdots & 0 \\
-d_{0} B_{2}^{-1} h_{2} & 0 & d_{2} & \cdots & 0 \\
\vdots & \vdots & \vdots & \ddots & \vdots \\
-d_{0} B_{p-1}^{-1} h_{p-1} & 0 & 0 & \cdots & d_{p-1}
\end{array}\right| \\
& =\frac{1}{1+h_{1}+\cdots+h_{p-1}}\left|\begin{array}{ccccc}
\nu & l-1 & l-1 & \cdots & l-1 \\
-d_{0} B_{1}^{-1} h_{1} & d_{1} & 0 & \cdots & 0 \\
-d_{0} B_{2}^{-1} h_{2} & 0 & d_{2} & \cdots & 0 \\
\vdots & \vdots & \vdots & \ddots & \vdots \\
-d_{0} B_{p-1}^{-1} h_{p-1} & 0 & 0 & \cdots & d_{p-1}
\end{array}\right|=0,
\end{aligned}
$$

where $\mu=\frac{l-1}{1+h_{1}+\cdots+h_{p-1}}, \nu=d_{0}\left(h_{1}+\cdots+h_{p-1}\right)+l-1 / A_{\ell}$ and

$$
d_{0}=\frac{A_{\ell}-1}{A_{\ell}}, d_{1}=\frac{A_{\ell}-B_{1}}{A_{\ell} B_{1}}, \ldots, d_{p-1}=\frac{A_{\ell}-B_{p-1}}{A_{\ell} B_{p-1}}
$$

The last determinant is linear with respect to $h_{1}, \ldots, h_{p-1}$ since these variables appear only in the first column as linear terms. By the cofactor expansion with respect to the first column, we can evaluate its coefficient of $h_{k}$ and its constant term. By multiplying $A_{\ell}^{p-1}\left(\prod_{i=1}^{p} A_{i}\right)\left(\prod_{j=1}^{p-1} B_{j}\right)$ to them, we have a linear equation

$$
\begin{aligned}
& -\sum_{k=1}^{p-1} B_{k}\left(A_{\ell}-1\right)\left(\prod_{j=1}^{j \neq \ell}\left(A_{\ell}-B_{j}\right)\right)\left(\prod_{1 \leq i \leq p}^{i \neq \ell} A_{i}-\prod_{1 \leq j \leq p-1}^{j \neq k} B_{j}\right) h_{k} \\
= & \left(\prod_{j=1}^{p-1}\left(A_{\ell}-B_{j}\right)\right)\left(\prod_{1 \leq i \leq p}^{i \neq \ell} A_{i}-\prod_{j=1}^{p-1} B_{j}\right)
\end{aligned}
$$


from $Q\left(1 / A_{\ell}\right)=0$. By letting $\ell$ vary from 1 to $p$, we have a system of linear equations with respect to $h_{1}, \ldots, h_{p-1}$. We can check that

$$
h_{k}=\frac{-\left(\prod_{1 \leq j \leq p-1}^{j \neq k}\left(B_{j}-1\right)\right)\left(\prod_{i=1}^{p}\left(A_{i}-B_{k}\right)\right)}{B_{k}\left(\prod_{1 \leq j \leq p-1}^{j \neq k}\left(B_{j}-B_{k}\right)\right)\left(\prod_{i=1}^{p}\left(A_{i}-1\right)\right)} \quad(1 \leq k \leq p-1)
$$

satisfy this system of linear equations. The uniqueness of $H$ completes this proposition.

Remark 3.1. Note that

$$
\mathrm{v} H^{t} \mathrm{v}=\operatorname{tr}(H)=\frac{\left(\prod_{i=1}^{p} A_{i}-\prod_{j=1}^{p-1} B_{j}\right) \prod_{j=1}^{p-1}\left(B_{j}-1\right)}{\prod_{i=1}^{p}\left(A_{i}-1\right) \prod_{j=1}^{p-1} B_{j}} .
$$

We have

$$
\frac{1-l}{\mathrm{vH}^{t} \mathrm{~V}}=\frac{\prod_{i=1}^{p}\left(A_{i}-1\right) \prod_{j=1}^{p-1} B_{j}}{\prod_{i=1}^{p} A_{i} \prod_{j=1}^{p-1}\left(B_{j}-1\right)}
$$

in which the factor $\prod_{i=1}^{p} A_{i}-\prod_{j=1}^{p-1} B_{j}$ in $1-l$ and $\mathrm{v} H^{t} \mathrm{v}$ is canceled.

We conclude this subsection by the following.

Theorem 3.1. Suppose the non-integral condition (9) for $a_{1}, \ldots, a_{p}$, $b_{1}, \ldots, b_{p-1}$. Then there exists a fundamental system $\mathbf{F}_{p}(x)$ of solutions to the hypergeometric differential equation (6) around $\dot{x}=\varepsilon$ such that the circuit matrices $M_{0}$ and $M_{1}$ along the loops $\rho_{0}$ and $\rho_{1}$ in (4) are expressed as

$$
M_{0}=\left(\begin{array}{cccc}
1 & & & \\
& B_{1}^{-1} & & \\
& & \ddots & \\
& & & B_{p-1}^{-1}
\end{array}\right), \quad M_{1}=i d_{p}-\frac{1-l}{\mathrm{v} H^{t} \mathrm{v}} H^{t} \mathrm{vv}
$$

where $\varepsilon$ is a sufficiently small positive real numbers, $A_{i}=e^{2 \pi \sqrt{-1} a_{i}}$ $(1 \leq i \leq p), B_{j}=e^{2 \pi \sqrt{-1} b_{j}}(1 \leq j \leq p-1), l=\left(\prod_{j=1}^{p-1} B_{j}\right) /\left(\prod_{i=1}^{p} A_{i}\right)$, 
$\mathrm{v}=(1, \ldots, 1)$ and

$$
\begin{aligned}
& H=\left(\begin{array}{cccc}
1 & & & \\
& h_{1} & & \\
& & \ddots & \\
& & & h_{p-1}
\end{array}\right) \\
& h_{k}=\frac{-\left(\prod_{1 \leq j \leq p-1}^{j \neq k}\left(B_{j}-1\right)\right)\left(\prod_{i=1}^{p}\left(A_{i}-B_{k}\right)\right)}{B_{k}\left(\prod_{1 \leq j \leq p-1}^{j \neq k}\left(B_{j}-B_{k}\right)\right)\left(\prod_{i=1}^{p}\left(A_{i}-1\right)\right)} \quad(1 \leq k \leq p-1) .
\end{aligned}
$$

\section{Monodromy Representation of $F_{C}$}

4.1. Lauricella's $F_{C}$ system. In this subsection, we refer to [AK], [HT] and [La]. Lauricella's hypergeometric series $F_{C}$ is defined by

$$
\begin{aligned}
& F_{C}\left(\begin{array}{c}
a_{1}, a_{2} \\
\left.b_{1}, \ldots, b_{m} ; x_{1}, \ldots, x_{m}\right)
\end{array}\right. \\
= & \sum_{n_{1}, \ldots, n_{m} \in \mathbb{N}^{m}} \frac{\left(a_{1}, n_{1}+\cdots+n_{m}\right)\left(a_{2}, n_{1}+\cdots+n_{m}\right)}{\left(b_{1}, n_{1}\right) \cdots\left(b_{m}, n_{m}\right)\left(1, n_{1}\right) \cdots\left(1, n_{m}\right)} x_{1}^{n_{1}} \cdots x_{m}^{n_{m}},
\end{aligned}
$$

where the vector $x=\left(x_{1}, \ldots, x_{m}\right)$ consisting of the main variables is in

$$
\left\{x \in \mathbb{C}^{m} \mid \sqrt{\left|x_{1}\right|}+\cdots+\sqrt{\left|x_{m}\right|}<1\right\},
$$

and $a_{1}, a_{2}, b_{1}, \ldots, b_{m}$ are complex parameters with $b_{1}, \ldots, b_{m} \notin-\mathbb{N}$. This series satisfies differential equations

$$
\begin{aligned}
& {\left[x_{i}\left(1-x_{i}\right) \partial_{i}^{2}-x_{i} \sum_{1 \leq j \leq m}^{j \neq i} x_{j} \partial_{i} \partial_{j}-\sum_{1 \leq j_{1}, j_{2} \leq m}^{j_{1} \neq i} x_{j_{1}} x_{j_{2}} \partial_{j_{1}} \partial_{j_{2}}\right.} \\
& \left.\quad+\left\{b_{i}-\left(a_{1}+a_{2}+1\right) x_{i}\right\} \partial_{i}-\left(a_{1}+a_{2}+1\right) \sum_{1 \leq j \leq m}^{j \neq i} x_{j} \partial_{j}-a_{1} a_{2}\right] f(x)=0,
\end{aligned}
$$

$(i=1, \ldots, m)$, which generate Lauricella's $F_{C}$ system of hypergeometric differential equations. Here $\partial_{i}$ is the partial differential operator with respect to $x_{i}$. Lauricella's $F_{C}$ system is integrable of rank $2^{m}$ and regular singular with singular locus

$$
S_{m}=\left\{x \in \mathbb{C}^{m} \mid x_{1} \cdots x_{m} R(X)=0\right\},
$$

where $R_{m}(x)$ is a polynomial of degree $2^{m-1}$ given by

$$
\prod_{\sigma_{1}, \ldots, \sigma_{m}= \pm 1}\left(1+\sigma_{1} \sqrt{x_{1}}+\cdots+\sigma_{m} \sqrt{x_{m}}\right) .
$$


Fact 4.1 ([La]). If $b_{1}, \ldots, b_{m} \notin \mathbb{Z}$ then a fundamental system of solutions to Lauricella's $F_{C}$ system around $\dot{x}=\left(\varepsilon_{1}, \ldots, \varepsilon_{m}\right)$ is given as follows:

\begin{tabular}{|c|c|}
\hline 1 & $F_{C}\left(\begin{array}{c}a_{1}, a_{2} \\
b_{1}, \ldots, b_{m}\end{array} ; x\right)$ \\
\hline$m$ & $\begin{array}{c}\vdots \\
x_{j}^{1-b_{j}} F_{C}\left(\begin{array}{c}a_{1}-b_{j}+1, a_{2}-b_{j}+1 \\
b_{1}, \ldots, 2-b_{j}, \ldots, b_{m}\end{array} ; x\right) \\
\vdots\end{array}$ \\
\hline$\vdots$ & $\vdots$ \\
\hline$\left(\begin{array}{c}m \\
r\end{array}\right)$ & {$\left[\prod_{j \in J_{r}} x_{j}^{1-b_{j}}\right] F_{C}\left(\begin{array}{c}a_{1}+\sum_{j \in J_{r}}\left(1-b_{j}\right), a_{2}+\sum_{j \in J_{r}}\left(1-b_{j}\right) \\
b_{1}+2 \delta_{1, J_{r}}\left(1-b_{1}\right), \ldots, b_{m}+2 \delta_{m, J_{r}}\left(1-b_{m}\right)\end{array} ; x\right)$} \\
\hline$\vdots$ & $\vdots$ \\
\hline 1 & {$\left[\prod_{j=1}^{m} x_{j}^{1-b_{j}}\right] F_{C}\left(\begin{array}{c}a_{1}+\sum_{j=1}^{m}\left(1-b_{j}\right), a_{2}+\sum_{j=1}^{m}\left(1-b_{j}\right) \\
2-b_{1}, \ldots, 2-b_{m}\end{array} ; x\right)$} \\
\hline
\end{tabular}

where $\varepsilon_{1}, \ldots, \varepsilon_{m}$ is a sufficiently small positive real numbers satisfying

$$
\varepsilon_{1} \gg \cdots \gg \varepsilon_{m}
$$

and $J_{r}$ is a subset of $\{1, \ldots, m\}$ of cardinality $r$, and

$$
\delta_{i, J_{r}}=\left\{\begin{array}{l}
1 \text { if } i \in J_{r}, \\
0 \quad \text { if } i \notin J_{r} .
\end{array}\right.
$$

We denote the solution with the factor $\prod_{j \in J_{r}} x_{j}^{1-b_{j}}$ in Fact 4.1 by $F_{C}^{J_{r}}(x)$. For the empty set $J_{0}=\phi$, we omit $J_{0}$ from this expression, i.e.,

$$
F_{C}^{J_{0}}(x)=F_{C}^{\phi}(x)=F_{C}\left(\begin{array}{c}
a_{1}, a_{2} \\
b_{1}, \ldots, b_{m}
\end{array} ; x\right) .
$$

4.2. Circuit matrices of Lauricella's $F_{C}$. In this subsection, we assume that

$$
b_{1}, \ldots, b_{m}, a_{1}-\sum_{j \in J} b_{j}, a_{2}-\sum_{j \in J} b_{j}, 2\left(a_{1}+a_{2}-\sum_{j=1}^{m} b_{j}\right) \notin \mathbb{Z},
$$

where $J$ runs over the subsets of $\{1, \ldots, m\}$. We set

$$
A_{i}=\exp \left(2 \pi \sqrt{-1} a_{i}\right)(i=1,2), \quad B_{j}=\exp \left(2 \pi \sqrt{-1} b_{j}\right)(1 \leq j \leq m) .
$$


We choose a fundamental system $\mathbf{F}_{C}^{g}(x)$ of solutions to Lauricella's system of $F_{C}$ around $\dot{x}=\left(\varepsilon_{1}, \ldots, \varepsilon_{m}\right)$ as

$$
\mathbf{F}_{C}^{g}(x)=g\left(\begin{array}{c}
F_{C}(x) \\
\vdots \\
F_{C}^{J}(x) \\
\vdots \\
F_{C}^{J_{m}}(x)
\end{array}\right), \quad g=\operatorname{diag}\left(g_{\phi}, \ldots, g_{J}, \ldots, g_{J_{m}}\right) \in G L_{2^{m}}(\mathbb{C})
$$

where $\operatorname{diag}\left(z_{1}, \ldots, z_{m}\right)$ denotes the diagonal matrix with diagonal entries $z_{1}, \ldots, z_{m}, J \subset\{1, \ldots, m\}$ are arranged lexicographically, i.e,

$$
J_{0}=\phi,\{1\},\{2\},\{1,2\},\{3\}, \ldots,\{1,2,3\},\{4\}, \ldots,\{1, \ldots, m\}=J_{m} .
$$

Note that the order of $J$ from the smallest is

$$
2^{J}=1+\sum_{i=1}^{m} \delta_{i, J} 2^{i-1}=1+\delta_{1, J} 2^{0}+\delta_{2, J} 2^{1}+\delta_{3, J} 2^{2}+\cdots+\delta_{m, J} 2^{m-1},
$$

where $\delta_{i, J}$ is given in (12).

Let $X$ be the complement of the singular locus $S_{m}$ in $\mathbb{C}^{m}$. Let $\rho$ be a loop in $X$ with base point $\dot{x}=\left(\varepsilon_{1}, \ldots, \varepsilon_{m}\right)$. Then there exists $M_{\rho} \in G L_{2^{m}}(\mathbb{C})$ such that the analytic continuation of $\mathbf{F}_{C}^{g}(x)$ along $\rho$ is expressed as $M_{\rho}^{g} \mathbf{F}_{C}^{g}(x)$. We call $M_{\rho}^{g}$ the circuit matrix of Lauricella's system $F_{C}$ with respect to the fundamental system $\mathbf{F}_{C}^{g}(x)$.

We give a system of generators of the fundamental group $\pi_{1}(X, \dot{x})$.

Fact $4.2([\mathrm{G}])$. Let $\rho_{i}(1 \leq i \leq m)$ be a loop defined by

$$
\rho_{i}:[0,1] \ni t \mapsto\left(\varepsilon_{1}, \ldots, \varepsilon_{i-1}, \quad \varepsilon_{i} e^{i-t h \sqrt{-1} t}, \quad \varepsilon_{i+1}, \ldots, \varepsilon_{m}\right) \in X,
$$

and let $\rho_{m+1}$ be a loop in the intersection of $X$ and the line

$$
L=\left\{\dot{x} \cdot t \in \mathbb{C}^{m} \mid t \in \mathbb{C}\right\}
$$

starting from $\dot{x}$, turning around the nearest point of the intersection $S_{m} \cap L$ to $\dot{x}$ once positively, and tracing back to $\dot{x}$. Then these loops generate the fundamental group $\pi_{1}(X, \dot{x})$, and satisfy the relations

$$
\rho_{j} \rho_{i}=\rho_{i} \rho_{j}, \quad\left(\rho_{i} \rho_{m+1}\right)^{2}=\left(\rho_{m+1} \rho_{i}\right)^{2}, \quad(1 \leq i<j \leq m) .
$$

Lemma 4.1. We have

$$
\begin{aligned}
\left(\rho_{m+1} \cdot \rho_{m} \cdot \rho_{m+1} \cdot \rho_{m}^{-1}\right) \cdot \rho_{m} & =\rho_{m} \cdot\left(\rho_{m+1} \cdot \rho_{m} \cdot \rho_{m+1} \cdot \rho_{m}^{-1}\right), \\
\rho_{m+1} \cdot \rho_{m} \cdot \rho_{m+1} \cdot \rho_{m}^{-1} & \stackrel{\widehat{X}}{\sim} \rho_{m}^{\prime},
\end{aligned}
$$

where $\rho_{m}^{\prime}$ is the generator of $\pi_{1}\left(X^{\prime}, \dot{x}^{\prime}\right)$ for $X^{\prime}=\mathbb{C}^{m-1}-S_{m-1}$ naturally embedded in the space $\widetilde{X}=\left\{x \in \mathbb{C}^{m} \mid x_{1} \cdots x_{m-1} R_{m}(x) \neq 0\right\}$ with 
base point $\dot{x}^{\prime}=\left(\varepsilon_{1}, \ldots, \varepsilon_{m-1}\right) \in X^{\prime}$, and $\stackrel{\widehat{X}}{\sim}$ denotes the homotopy equivalence in $\widehat{X}$.

Proof. It is a direct consequence from Fact 4.2 that $\rho_{m+1} \cdot \rho_{m} \cdot \rho_{m+1} \cdot \rho_{m}^{-1}$ commutes with $\rho_{m}$. Let the line $L$ move along $\rho_{m}$. By tracing the deformation of $\rho_{m+1}$, we have a loop starting from $\dot{x}$, turning around the second nearest point $S_{m} \cap L$ to $\dot{x}$ once positively, and tracing back to $\dot{x}$. Since the base point $\dot{x}$ moves along $\rho_{m}$, this deformation is homotopic to $\rho_{m} \cdot \rho_{m+1} \cdot \rho_{m}^{-1}$. Thus the loop

$$
\rho_{m+1} \cdot\left(\rho_{m} \cdot \rho_{m+1} \cdot \rho_{m}^{-1}\right)
$$

turns around the first and second nearest points $S_{m} \cap L$ to $\dot{x}$ once positively. Consider the limit as $x_{m} \rightarrow 0$. These points meets and the polynomial $R_{m}\left(x_{1}, \ldots, x_{m}\right)$ reduces to $R_{m-1}\left(x_{1}, \ldots, x_{m-1}\right)^{2}$. Moreover the duplicated point is the nearest point of the intersection $S_{m-1} \cap L^{\prime}$ to $\dot{x}^{\prime}$. Hence the loop $\rho_{m+1} \cdot \rho_{m} \cdot \rho_{m+1} \cdot \rho_{m}^{-1}$ is homotopic to $\rho_{m}^{\prime}$.

We set

$$
M_{i}^{g}=M_{\rho_{i}}^{g} \quad(1 \leq i \leq m+1) .
$$

Lemma 4.2. The circuit matrix $M_{i}^{g}$ of Lauricella's system $F_{C}$ is a diagonal matrix whose entry corresponding to a subset $J$ of $\{1, \ldots, m\}$ is

$$
B_{i}^{-\delta_{i, J}}=\left\{\begin{array}{ccc}
\frac{1}{B_{i}} & \text { if } & i \in J, \\
1 & \text { if } & i \notin J,
\end{array}\right.
$$

where $B_{i}=\exp \left(2 \pi \sqrt{-1} b_{i}\right)$. They are independent of the diagonal matrix $g \in G L_{2^{m}}(\mathbb{C})$.

Proof. We have only to note that the solution $F_{C}^{J}$ has a factor $x_{i}^{1-b_{i}}$ if and only if $i \in J$.

There are $2^{m-1}$ subsets $J$ 's such that $i \in J$ for any $1 \leq i \leq m$. The both eigen spaces of $M_{i}$ of eigenvalue $1 / B_{i}$ and of eigenvalue 1 are $2^{m-1}$ dimensional.

We need the following two facts given in $[\mathrm{G}]$.

Fact 4.3. Let $M_{\rho}^{g}$ be the circuit matrix along $\rho \in \pi_{1}(X, \dot{x})$ with respect to $\mathbf{F}_{C}^{g}(x)$. Then there exists a diagonal matrix $H \in G L_{2^{m}}(\mathbb{C})$ such that

$$
M_{\rho}^{g} H^{t}\left(M_{\rho}^{g}\right)^{\vee}=H
$$

where $z\left(a_{1}, a_{2}, b_{1}, \ldots, b_{m}\right)^{\vee}=z\left(-a_{1},-a_{2},-b_{1}, \ldots,-b_{m}\right)$ for any function $z$ of the parameters. 
Note that the matrix $H$ depends on the diagonal matrix $g \in G L_{2^{m}}(\mathbb{C})$. We treat the entries of $H$ as indeterminants.

Fact 4.4. The eigenvalues of the circuit matrix $M_{m+1}^{g}$ consists of 1 and $l$. The eigenspace of eigenvalue $l$ is spanned by a row vector $v$. The eigenspace of eigenvalue 1 is $2^{m}-1$ dimensional.

Remark 4.1. It is shown in $[\mathrm{G}]$ that the eigenvalue $l$ of the circuit matrix $M_{m+1}$ is

$$
(-1)^{m+1}\left(\prod_{j=1}^{m} B_{j}\right) /\left(A_{1} A_{2}\right),
$$

which is different from 1 under our assumption, where $A_{i}=\exp \left(2 \pi \sqrt{-1} a_{i}\right)$ $(i=1,2)$. In this subsection, we treat $\lambda$ as an indeterminant different from 1, and we show that $\lambda$ should take the above value.

Lemma 4.3. Let $v=\left(\ldots, v_{J_{r}}, \ldots\right)$ be an eigenvector of $M_{m+1}^{g}$ of eigenvalue $l$. Then the eigenspace of $M_{m+1}^{g}$ of eigenvalue 1 is characterized as

$$
\left\{w \in \mathbb{C}^{2^{m}} \mid w H^{t} v^{\vee}=0\right\} .
$$

Moreover, the vector $v$ satisfies

$$
v H^{t} v^{\vee} \neq 0 .
$$

Proof. Trace the proof of Lemma 2.4.

Lemma 4.4. Let $v$ be an eigenvector of $M_{m+1}^{g}$ of eigenvalue $l$. Then the circuit matrix $M_{m+1}^{g}$ is expressed as

$$
M_{m+1}^{g}=i d_{2^{m}}-\frac{1-l}{v H^{t} v^{\vee}} H^{t} v^{\vee} v
$$

Moreover, no entry of $v$ vanishes.

Proof. For the expression of $M_{m+1}^{g}$, trace the proof of Lemma 3.4. We show that the $j$-th entry $v_{j}$ of $v$ does not vanish. Under our assumption (13), Lauricella's $F_{C}$ system is irreducible by Theorem 13 in [HT]. Suppose that $v_{j}=0$. Then the matrix $M_{m+1}^{g}$ takes the form

$$
j\left(\begin{array}{ccc}
* & j & \\
& { }^{t} \mathbf{0} & * \\
\mathbf{0} & 1 & \mathbf{0}^{\prime} \\
* & { }^{t} \mathbf{0}^{\prime} & *
\end{array}\right)
$$

by its expression, where $\mathbf{0}$ and $\mathbf{0}^{\prime}$ are zero vectors. Since $M_{i}^{g}(1 \leq$ $i \leq m$ ) are diagonal, the space spanned by the $j$-th unit vector is invariant under the actions of circuit matrices. This contradicts to the irreducibility of the system. Therefore, we have $v_{j} \neq 0$. 
We choose $g \in G L_{2^{m}}(\mathbb{C})$ so that the eigenvector of eigenvalue $l$ becomes $\mathrm{v}=(1, \ldots, 1)$. From now on, we fix the entries of $g$ as above values. We denote the fundamental system of solutions to Lauricella's $F_{C}$ around $\dot{x}$ for this $g$ in $\mathbf{F}_{C}^{g}(x)$ by $\mathbf{F}_{C}(x)$. We denote the circuit matrices with respect to $\mathbf{F}_{C}(x)$ by $M_{1}, \ldots, M_{m}$ and $M_{m+1}$. Explicit forms of $M_{1}, \ldots, M_{m}$ are given in Lemma 4.2, and we have

$$
M_{m+1}=i d_{2^{m}}-\frac{1-l}{\mathrm{v} H^{t} \mathrm{v}} H^{t} \mathrm{vv}
$$

where we regard $l$ and the entries of $H$ as indeterminants. By evaluating them, we determine the expression of $M_{m+1}$. By a scalar multiplication to $H$, we can assume that

$$
H=\operatorname{diag}\left(1, \ldots, h_{J}, \ldots\right),
$$

where $J$ runs over the non-empty subsets of $\{1, \ldots, m\}$ arranged lexicographically. Note that the matrix $H$ is unique after this normalization.

Lemma 4.5. The eigenspace of $M_{m+1}$ of eigenvalue 1 is spanned by row vectors

$$
h_{J} e_{\phi}-e_{J}, \quad \phi \neq J \subset\{1, \ldots, m\},
$$

where $e_{\phi}=(1,0, \ldots, 0) \in \mathbb{N}^{2^{m}}$ and $e_{J}$ is the $2^{J}$-th unit vector of size $2^{m}$.

Proof. Since $\mathrm{v}=(1, \ldots, 1)$, and $H=\operatorname{diag}\left(1, \ldots, h_{J}, \ldots\right)$, we have

$$
\left(h_{J} e_{\phi}-e_{J}\right) H^{t} \mathrm{v}^{\vee}=\left(h_{J} e_{\phi}-h_{J} e_{J}\right)^{t} \mathrm{v}=h_{J}-h_{J}=0 .
$$

By Lemma 4.3, these vectors span the the eigenspace of $M_{m+1}$ of eigenvalue 1.

Proposition 4.1. We have

$$
\begin{aligned}
h_{J} & =(-1)^{|J|} \frac{\left(A_{1}-\prod_{j \in J} B_{j}\right)\left(A_{2}-\prod_{j \in J} B_{j}\right)}{\left(A_{1}-1\right)\left(A_{2}-1\right) \prod_{j \in J} B_{j}}, \\
\operatorname{tr}(H) & =\frac{\left(A_{1} A_{2}+(-1)^{m} \prod_{j=1}^{m} B_{j}\right) \prod_{j=1}^{m}\left(B_{j}-1\right)}{\left(A_{1}-1\right)\left(A_{2}-1\right) \prod_{j=1}^{m} B_{j}}, \\
\lambda & =(-1)^{m+1}\left(\prod_{j=1}^{m} B_{j}\right) /\left(A_{1} A_{2}\right),
\end{aligned}
$$

where $|J|$ is the cardinality of $J$. 
Proof. At first, we determine the entries of $H$. We use the induction on $m$. We have shown in Proposition 2.1 that our assertion holds for $m=1$.

Assume that our assertion holds for $m-1$. From our fundamental system $\mathbf{F}_{C}(x)$ to Lauricella's system $F_{C}$, we choose the $2^{m-1}$ solutions corresponding to the subsets of $\{1, \ldots, m-1\}$ and restrict to the hyperplane $x_{m}=0$. Then we have the fundamental system $\mathbf{F}_{C}^{\prime}(x)$ to Lauricella's system $F_{C}$ of the $m-1$ variables $x_{1}, \ldots, x_{m-1}$. Note that the top-left block matrix of $M_{i}(1 \leq i \leq m-1)$ of size $2^{m-1}$ coincides with the circuit matrix $M_{i}^{\prime}$ for this fundamental system $\mathbf{F}_{C}^{\prime}(x)$. By Lemma 4.1, the matrix $M_{m+1} M_{m} M_{m+1} M_{m}^{-1}$ commutes with $M_{m}$. Thus it is block diagonal with block size $2^{m-1}$, i.e.,

$$
M_{m+1} M_{m} M_{m+1} M_{m}^{-1}=\left(\begin{array}{cc}
M_{m}^{\prime} & O \\
O & M_{m}^{\prime \prime}
\end{array}\right) .
$$

We consider its top-left block matrix $M_{m}^{\prime}$ of size $2^{m-1}$. By Lemma 4.1, this can be regarded as the circuit matrix of $\rho_{m}^{\prime} \in \pi_{1}\left(X^{\prime}, \dot{x}^{\prime}\right)$ with respect to the restriction of chosen $2^{m-1}$ solutions to $x_{m}=0$. The eigenspace of $M_{m}^{\prime}$ of eigenvalue 1 is $2^{m-1}-1$ dimensional by Fact 4.4 . By the assumption of the induction, the other eigenvalue of $M_{m}^{\prime}$ is $l^{\prime}=(-1)^{m}\left(\prod_{j=1}^{m-1} B_{j}\right) /\left(A_{1} A_{2}\right)$. We show that $\mathrm{v}^{\prime}=(1, \ldots, 1) \in \mathbb{N}^{2^{m-1}}$ is its eigenvector. This is equivalent to show that the top-left block of the normalizing matrix $g \in G L_{2^{m}}(\mathbb{C})$ coincides with the normalizing matrix $g^{\prime} \in G L_{2^{m-1}}(\mathbb{C})$ for the $m-1$ variables case modulo non-zero scalar multiplication. Let $e_{J^{\prime}}$ and $e_{J^{\prime}}^{\prime}$ be the $e^{J^{\prime}}$-th unit vector of size $2^{m}$ and that of size $2^{m-1}$ for a subset $J^{\prime}$ of $\{1, \ldots, m-1\}$. Then we have $e_{J^{\prime}} M_{m}=e_{J^{\prime}}$ by Lemma 4.2. Lemma 4.5 yields that

$$
\left(h_{J^{\prime}} e_{\phi}-e_{J^{\prime}}\right) M_{m+1} M_{m} M_{m+1} M_{m}^{-1}=h_{J^{\prime}} e_{\phi}-e_{J^{\prime}}
$$

for any non-empty set $J^{\prime}$ of $\{1, \ldots, m-1\}$. Thus

$$
h_{J^{\prime}} e_{\phi}^{\prime}-e_{J^{\prime}}^{\prime} \quad\left(\phi \neq J^{\prime} \subset\{1, \ldots, m-1\}\right)
$$

span the eigenspace of $M_{m}^{\prime}$ of eigenvalue 1 . Since

$$
\left(h_{J^{\prime}} e_{\phi}^{\prime}-e_{J^{\prime}}^{\prime}\right) H^{\prime} \mathrm{v}^{\prime}=0
$$

for the top-left block matrix $H^{\prime}$ of $H$ of size $2^{m-1}, \mathrm{v}^{\prime}$ is an eigenvector of $M_{m}^{\prime}$ of eigenvalue $l^{\prime}$ by Lemma 4.3. Hence $H^{\prime}$ coincides with the matrix for the case of $m-1$ variables, i.e., $h_{J^{\prime}}$ for any subset of $\{1, \ldots, m-1\}$ 
should be equal to

$$
(-1)^{\left|J^{\prime}\right|} \frac{\left(A_{1}-\prod_{j \in J^{\prime}} B_{j}\right)\left(A_{2}-\prod_{j \in J^{\prime}} B_{j}\right)}{\left(\prod_{j \in J^{\prime}} B_{j}\right)\left(A_{1}-1\right)\left(A_{2}-1\right)} .
$$

From our fundamental system $\mathbf{F}_{C}(x)$ to Lauricella's system $F_{C}$, we choose the $2^{m-1}$ solutions corresponding to the subsets of $\{1, \ldots, m-$ $2, m\}$ and restrict to the hyperplane $x_{m-1}=0$. Then we can lead $h_{J^{\prime}}$ for any subset $J^{\prime}$ of $\{1, \ldots, m-2, m\}$ similarly to the previous way by the symmetry of the Lauricella' system $F_{C}$. Especially, we have

$$
h_{m}=-\frac{\left(A_{1}-B_{m}\right)\left(A_{2}-B_{m}\right)}{\left(A_{1}-1\right)\left(A_{2}-1\right) B_{m}} .
$$

From our fundamental system $\mathbf{F}_{C}(x)$ to Lauricella's system $F_{C}$, we choose the $2^{m-1}$ solutions corresponding to the subsets of $\{1, \ldots, m\}$ including the index $m$. Note that these solutions include the factor $x_{m}^{1-b_{m}}$. We consider the ratio of them and restrict it to $x_{m}=0$. This restriction of the ratio coincides with the ratio of the fundamental system $\mathbf{F}_{C}(x)$ to Lauricella's system $F_{C}$ of the $m-1$ variables $x_{1}, \ldots, x_{m-1}$ with parameters $a_{1}-b_{m}, a_{2}-b_{m}, b_{1}, \ldots, b_{m-1}$ by Fact 4.1. Its circuit matrices appear in the bottom-right blocks of $M_{i}(1 \leq i \leq m-1)$ and of $M_{m+1} M_{m} M_{m+1} M_{m}^{-1}$. We can show similarly to the previous that $\mathrm{v}^{\prime}=(1, \ldots, 1) \in \mathbb{N}^{2^{m-1}}$ is an eigenvector of the bottom-right block matrix $M_{m}^{\prime \prime}$ of $M_{m+1} M_{m} M_{m+1} M_{m}^{-1}$ of non-one eigenvalue. By the assumption of the induction, for any subset $J^{\prime}$ of $\{1, \ldots, m-1\}$, the ratio of $h_{J^{\prime} \cup\{m\}}$ and $h_{m}$ coincides with $\left.h_{J^{\prime}}\right|_{\left(A_{1}, A_{2}\right) \rightarrow\left(A_{1} / B_{m}, A_{2} / B_{m}\right)}$, which is the transformed $h_{J^{\prime}}$ by the replacement

$$
\left(A_{1}, A_{2}\right) \rightarrow\left(A_{1} / B_{m}, A_{2} / B_{m}\right)
$$

Hence we have

$$
\begin{aligned}
& h_{J^{\prime} \cup\{m\}}=\left.h_{m} \cdot h_{J^{\prime}}\right|_{\left(A_{1}, A_{2}\right) \rightarrow\left(A_{1} / B_{m}, A_{2} / B_{m}\right)} \\
= & -\frac{\left(A_{1}-B_{m}\right)\left(A_{2}-B_{m}\right)}{\left(A_{1}-1\right)\left(A_{2}-1\right) B_{m}} \cdot(-1)^{\left|J^{\prime}\right|} \frac{\left(\frac{A_{1}}{B_{m}}-\prod_{j \in J^{\prime}} B_{j}\right)\left(\frac{A_{2}}{B_{m}}-\prod_{j \in J^{\prime}} B_{j}\right)}{\left(\frac{A_{1}}{B_{m}}-1\right)\left(\frac{A_{2}}{B_{m}}-1\right) \prod_{j \in J^{\prime}} B_{j}} \\
= & (-1)^{\left|J^{\prime} \cup\{m\}\right|} \frac{\left(A_{1}-\prod_{j \in J^{\prime} \cup\{m\}} B_{j}\right)\left(A_{2}-\prod_{j \in J^{\prime} \cup\{m\}} B_{j}\right)}{\left(A_{1}-1\right)\left(A_{2}-1\right) \prod_{j \in J^{\prime} \cup\{m\}} B_{j}} .
\end{aligned}
$$


Next we compute the trace of $H$. We have seen that our assertion on $\operatorname{tr}(H)$ holds for $m=1$ in Remark 2.1. Suppose that our assertion on $\operatorname{tr}(H)$ holds for $m-1$. Let $H^{\prime}$ be the top-left block matrix of $H$ of size $2^{m-1}$. By the previous consideration and the assumption of the induction, we have

$$
\begin{aligned}
& \operatorname{tr}(H)=\operatorname{tr}\left(H^{\prime}\right)+\left.h_{m} \cdot \operatorname{tr}\left(H^{\prime}\right)\right|_{\left(A_{1}, A_{2}\right) \rightarrow\left(A_{1} / B_{m}, A_{2} / B_{m}\right)} \\
= & \frac{\left(A_{1} A_{2}+(-1)^{m-1} \prod_{j=1}^{m-1} B_{j}\right) \prod_{j=1}^{m-1}\left(B_{j}-1\right)}{\left(A_{1}-1\right)\left(A_{2}-1\right) \prod_{j=1}^{m-1} B_{j}} \\
& -\frac{\left(A_{1}-B_{m}\right)\left(A_{2}-B_{m}\right)}{\left(A_{1}-1\right)\left(A_{2}-1\right) B_{m}} \cdot \frac{\left(\frac{A_{1} A_{2}}{B_{m}^{2}}+(-1)^{m-1} \prod_{j=1}^{m-1} B_{j}\right) \prod_{j=1}^{m-1}\left(B_{j}-1\right)}{\left(\frac{A_{1}}{B_{m}}-1\right)\left(\frac{A_{2}}{B_{m}}-1\right) \prod_{j=1}^{m-1} B_{j}} .
\end{aligned}
$$

By taking out the common factor

$$
\prod_{j=1}^{m-1}\left(B_{j}-1\right) /\left[\left(A_{1}-1\right)\left(A_{2}-1\right) \prod_{j=1}^{m} B_{j}\right]
$$

from the above, we have

$$
\begin{aligned}
& \left(A_{1} A_{2} B_{m}+(-1)^{m-1} \prod_{j=1}^{m} B_{j}\right)-\left(A_{1} A_{2}+(-1)^{m-1} B_{m} \prod_{j=1}^{m} B_{j}\right) \\
= & \left(A_{1} A_{2}+(-1)^{m} \prod_{j=1}^{m} B_{j}\right)\left(B_{m}-1\right),
\end{aligned}
$$

which yields our assertion on $\operatorname{tr}(H)$ for $m$.

Finally, we determine the eigenvalue $l$ so that $u=(1, \ldots, 1,0, \ldots, 0)$ is an eigenvector of $M_{m+1} M_{m} M_{m+1} M_{m}^{-1}$. Note that

$$
M_{m} M_{m+1} M_{m}^{-1}=i d_{2^{m}}-\frac{1-l}{w H^{t} w^{\vee}} H^{t} w^{\vee} w
$$

for $w=v M_{m}^{-1}=\left(1, \ldots, 1, B_{m}, \ldots, B_{m}\right)$. Note also that

$$
\mathrm{v} H^{t} \mathrm{v}=w H^{t} w^{\vee}=\operatorname{tr}(H), \quad u H^{t} \mathrm{v}=u H^{t} w^{\vee}=\operatorname{tr}\left(H^{\prime}\right)
$$




$$
\begin{aligned}
& \mathrm{v} H^{t} w^{\vee}= \operatorname{tr}\left(H^{\prime}\right)+\left.B_{m}^{-1} h_{m} \operatorname{tr}\left(H^{\prime}\right)\right|_{\left(A_{1}, A_{2}\right) \rightarrow\left(A_{1} / B_{m}, A_{2} / B_{m}\right)} \\
&= \frac{A_{1} A_{2}\left(B_{m}+1\right) \prod_{j=1}^{m}\left(B_{j}-1\right)}{\left(A_{1}-1\right)\left(A_{2}-1\right) B_{m} \prod_{j=1}^{m} B_{j}}, \\
& \frac{\mathrm{v} H^{t} w^{\vee}}{\mathrm{v} H^{t} \mathrm{v}}=\frac{A_{1} A_{2}\left(B_{m}+1\right)}{\left(A_{1} A_{2}+(-1)^{m} \prod_{j=1}^{m} B_{j}\right) B_{m}} .
\end{aligned}
$$

Thus we have

$$
\begin{aligned}
& u M_{m+1} M_{m} M_{m+1} M_{m}^{-1} \\
= & u\left(i d_{2^{m}}-\frac{1-l}{\mathrm{v} H^{t} \mathrm{v}} H^{t} \mathrm{vv}\right)\left(i d_{2^{m}}-\frac{1-l}{w H^{t} w^{\vee}} H^{t} w^{\vee} w\right) \\
= & u-\frac{(1-l) u H^{t} \mathrm{v}}{\mathrm{v} H^{t} \mathrm{v}} \mathrm{v}-\frac{(1-l) u H^{t} \mathrm{v}}{\mathrm{v} H^{t} \mathrm{v}} w+\frac{(1-l)^{2}\left(u H^{t} \mathrm{v}\right)\left(\mathrm{v} H^{t} w^{\vee}\right)}{\left(\mathrm{v} H^{t} \mathrm{v}\right)^{2}} w \\
= & u-\frac{u H^{t} \mathrm{v}}{\mathrm{v}^{t}{ }^{t} \mathrm{v}}(1-l)(\mathrm{v}+w)+\frac{\left(u H^{t} \mathrm{v}\right)\left(\mathrm{v} H^{t} w^{\vee}\right)}{\left(\mathrm{v} H^{t} \mathrm{v}\right)^{2}}(1-l)^{2} w,
\end{aligned}
$$

which should be a scalar multiple of $u$. Since its $2^{m}$ entry vanishes, $l$ satisfies the quadratic equation

$$
\left(1+B_{m}\right)(1-l)=\frac{\mathrm{v} H^{t} w^{\vee}}{\mathrm{v} H^{t} \mathrm{v}} B_{m}(1-l)^{2}
$$

Hence we have

$$
1-l=\frac{\left(B_{m}+1\right) \mathrm{v} H^{t} \mathrm{v}}{B_{m} \mathrm{v} H^{t} w^{\vee}}=1+(-1)^{m}\left(\prod_{j=1}^{m} B_{j}\right) /\left(A_{1} A_{2}\right),
$$

under the assumption $l \neq 1$.

Remark 4.2. It is easy to obtain

$$
l= \pm\left(\prod_{j=1}^{m} B_{j}\right) /\left(A_{1} A_{2}\right)
$$

In fact, the determinant of $M_{m+1} M_{m} M_{m+1} M_{m}^{-1}$ is $l^{2}$. On the other hand, the determinants of its top-left block matrix and bottom-right 
one are

$$
\begin{aligned}
\operatorname{det}\left(M_{m}^{\prime}\right) & =(-1)^{m}\left(\prod_{j=1}^{m-1} B_{j}\right) /\left(A_{1} A_{2}\right), \\
\left.\operatorname{det}\left(M_{m}^{\prime}\right)\right|_{\left(A_{1}, A_{2}\right) \rightarrow\left(A_{1} / B_{m}, A_{2} / B_{m}\right)} & =(-1)^{m} \prod_{j=1}^{m-1} B_{j} /\left[\left(A_{1} / B_{m}\right)\left(A_{2} / B_{m}\right)\right],
\end{aligned}
$$

respectively. These product is equal to $l^{2}$.

Remark 4.3. We have

$$
\frac{1-l}{\mathrm{v} H^{t} \mathrm{~V}}=\frac{\left(A_{1}-1\right)\left(A_{2}-1\right) \prod_{j=1}^{m} B_{j}}{A_{1} A_{2} \prod_{j=1}^{m}\left(B_{j}-1\right)},
$$

in which the factor $A_{1} A_{2}+(-1)^{m} \prod_{j=1}^{m} B_{j}$ in $1-l$ and $\mathrm{v} H^{t} \mathrm{v}$ is canceled.

Theorem 4.1. Suppose the non-integral condition (13) for $a_{1}, a_{2}$ and $b_{1}, \ldots, b_{m}$. Then there exists a fundamental system $\mathbf{F}_{C}(x)$ of solutions to Lauricella's $F_{C}$ system around $\dot{x}=\left(\varepsilon_{1}, \ldots, \varepsilon_{m}\right)$ such that the circuit matrices $M_{1}, \ldots, M_{m}$ and $M_{m+1}$ along the loops $\rho_{1}, \ldots, \rho_{m}$ and $\rho_{m+1}$ in Fact 4.2 are expressed as

$$
\begin{aligned}
M_{i} & =\operatorname{diag}\left(1, \ldots, B_{i}^{-\delta_{i, J}}, \ldots\right) \quad(1 \leq i \leq m), \\
M_{m+1} & =i d_{2^{m}}-\frac{1-l}{\mathrm{v} H^{t} \mathrm{~V}} H^{t} \mathrm{vv},
\end{aligned}
$$

where $\varepsilon$ is a sufficiently small positive real numbers, $A_{i}=e^{2 \pi \sqrt{-1} a_{i}}$ $(i=1,2), B_{j}=e^{2 \pi \sqrt{-1} b_{j}}(1 \leq j \leq m), \mathrm{v}=(1, \ldots, 1) \in \mathbb{N}^{2^{m}}$,

$$
\begin{aligned}
& \delta_{i, J}= \begin{cases}1 & \text { if } \quad i \in J, \\
0 & \text { if } i \notin J,\end{cases} \\
& H=\operatorname{diag}\left(1, \ldots, h_{J}, \ldots\right) \text {, } \\
& h_{J}=(-1)^{|J|} \frac{\left(A_{1}-\prod_{j \in J} B_{j}\right)\left(A_{2}-\prod_{j \in J} B_{j}\right)}{\left(\prod_{j \in J} B_{j}\right)\left(A_{1}-1\right)\left(A_{2}-1\right)}, \\
& \lambda=(-1)^{m+1}\left(\prod_{j=1}^{m} B_{j}\right) /\left(A_{1} A_{2}\right),
\end{aligned}
$$

$J$ runs over the non-empty subsets of $\{1, \ldots, m\}$ arranged lexicographically, and $|J|$ is the cardinality of $J$. 
Remark 4.4. We have seen that $N_{m}=M_{m+1} M_{m} M_{m+1} M_{m}^{-1}$ is block diagonal with block size $2^{m-1}$. We inductively define matrices $N_{m-k}$ as

$$
N_{m-k}=N_{m-k+1} M_{m-k} N_{m-k+1} M_{m-k}^{-1}, \quad k=1, \ldots, m-2 .
$$

Then the matrix $N_{m-k}$ is block diagonal with block size $2^{m-k-1}$.

\section{REFERENCES}

[AK] Appell P. and Kampé de Fériet M. J., Fonctions hypergéométriques et hypersphériques: polynomes d'Hermite, Gauthier-Villars, Paris, 1926.

[BH] Beukers F. and Heckman G., Monodromy for the hypergeometric function ${ }_{n} F_{n-1}$, Invent. math., 95 (1989), 325-354.

[G] Goto Y., The monodromy representation for Lauricella's hypergeometric function $F_{C}$, preprint 2014, arXiv:1403.1654 2 .

[GM] Goto Y. and Matsumoto K., The monodromy representation and twisted period relations for Appell's hypergeometric function $F_{4}$, to appear in Nagoya M. J..

[HT] Hattori R. and Takayama N., The singular locus of Lauricella's $F_{C}, J$. Math. Soc. Japan, 66 (2014), 981-995.

[HU] Haraoka Y. and Ueno Y., Rigidity for Appellfs hypergeometric series $F_{4}$, Funkcial. Ekvac., 51 (2008), 149-164.

[IKSY] Iwasaki K., Kimura H., Shimomura S. and Yoshida M., From Gauss to Painlevé, Vieweg, Braunschweig, Wiesbaden, 1991.

[Kan] Kaneko J., Monodromy group of Appell's system $\left(F_{4}\right)$, Tokyo J. Math., 4 (1981), 35-54.

[Kat] Kato M., Connection formulas for Appell's system $F_{4}$ and some applications, Funkcial. Ekvac., 38 (1995), 243-266.

[La] Lauricella G., Sulle funzioni ipergeometriche a più variabili, Rend. Circ. Mat. Palermo , 7 (1893), 111-158.

[Le] Levelt, A. H. M., Hypergeometric functions III, Nederl. Akad. Wetensch. Proc. Ser. A 64, 23 (1961), 386-396.

[Ma] Matsumoto K., Monodromy and Pfaffian of Lauricella's $F_{D}$ in terms of the intersection forms of twisted (co)homology groups, Kyushu J. Math., 67 (2013), $367-387$.

[MY] Matsumoto K. and Yoshida M., Monodromy of Lauricella's hypergeometric

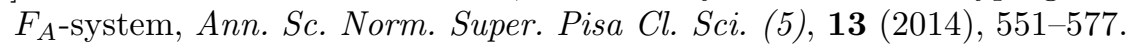

[Mi] Mimachi, K., Intersection numbers for twisted cycles and the connection problem associated with the generalized hypergeometric function ${ }_{n+1} F_{n}$, Int. Math. Res. Not. IMRN, (2011), 1757-1781.

[O] Ohara K., Computation of the monodromy of the generalized hypergeometric function ${ }_{p} F_{p-1}\left(a_{1}, \ldots, a_{p} ; b_{2}, \ldots, b_{p} ; z\right)$, Kyushu J. Math., 51 (1997), 101-124.

[T] Takano K., Monodromy group of the system for Appell's $F_{4}$ Funkcial. Ekvac., 23 (1980), 97-122.

E-mail address: matsu@math.sci.hokudai.ac.jp

(Matsumoto) Department of Mathematics, Hokkaido University, SapPORO 060-0810, JAPAN 